\title{
Synthesis and Characterization of Silver Nanoparticle-Multiwalled Carbon Nanotube Composites
}

\author{
Dunieskys G. Larrude, Marcelo E. H. Maia da Costa, and Fernando L. Freire Jr. \\ Physics Department, Pontifical Catholic University of Rio de Janeiro, 22451-900 Rio de Janeiro, RJ, Brazil \\ Correspondence should be addressed to Fernando L. Freire Jr.; lazaro.puc@gmail.com
}

Received 19 January 2014; Revised 12 March 2014; Accepted 23 April 2014; Published 12 May 2014

Academic Editor: Claude Estournès

Copyright (C) 2014 Dunieskys G. Larrude et al. This is an open access article distributed under the Creative Commons Attribution License, which permits unrestricted use, distribution, and reproduction in any medium, provided the original work is properly cited.

Multiwalled carbon nanotubes (MWCNTs) grown by spray pyrolysis have been decorated with silver nanoparticles prepared via the silver mirror reaction. Good dispersion of silver nanostructures was obtained on the surface of MWCNTs, resulting in an efficient and simple wet chemistry method for increasing the reactivity of the carbon nanotubes surfaces. High-resolution transmission electron microscopy showed the orientations of the crystallography planes of the anchored silver nanoparticles and revealed their size distribution. Raman spectroscopy results confirm that the composite material preserves the integrity of the MWCNTs. Scanning electron microscopy, X-ray diffraction, and X-ray photoelectron spectroscopy were also employed for sample characterization.

\section{Introduction}

During the last decades, extensive research has led to the development of versatile methods for modifying carbon nanotubes (CNTs) and to obtain derivatives with more attractive features [1-8]. To this end, CNTs decorated with metal nanoparticles (NPs), which exhibit outstanding chemical activity due to their large active surface area and crystallographic surface structure, have been examined for potentials applications in nanoelectronics and heterogeneous catalysis as well as chemical and biochemical sensors [915]. Because nanoparticles are notably different from their bulk materials with respect to their electronic, optical, and catalytic properties originating from their small dimensions [16], one can alter and/or enhance their intrinsic properties by controlling the size and shape as well as the distribution of metal nanoparticles on carbon nanotubes.

Several methodologies have been developed to obtain silver nanoparticle-CNT composites, such as solid-state reaction, thermal evaporation, surface chemical reduction of the metal salt, and wet chemical routes $[10-14,17-20]$ promoting either covalent or noncovalent functionalization between NPs and CNTs. For sensing applications, functionalization of CNTs sidewalls can improve the excellent potential of carbon nanotubes as sensitive material for detecting very low levels of chemical or biological molecules, enhancing the interaction between a specific chemical species and the nanotubes, as well as the selectivity of the adsorption process [21-23]. Silver nanoparticles can be prepared via a simple and inexpensive technique (i.e., the silver mirror reaction) [24]. Herein, multiwalled carbon nanotubes (MWCNTs) synthesized by the spray pyrolysis method using toluene and ferrocene precursors were decorated with silver nanoparticles following a similar chemical route. A detailed investigation is presented. High-resolution transmission electron microscopy (HRTEM) revealed the orientations of the crystallography planes of the anchored silver nanoparticles. This information can be important for modeling the process of nucleation and growth of the nanoparticles on the nanotubes surfaces. Raman spectroscopy, scanning electron microscopy (SEM), $\mathrm{X}$-ray diffraction (XRD), and X-ray photoelectron spectroscopy (XPS) were also employed for sample characterization.

\section{Materials and Methods}

2.1. Synthesis of the Composites. The multiwalled carbon nanotubes were synthesized using the spray pyrolysis method as previously reported elsewhere [26]. This method has the 


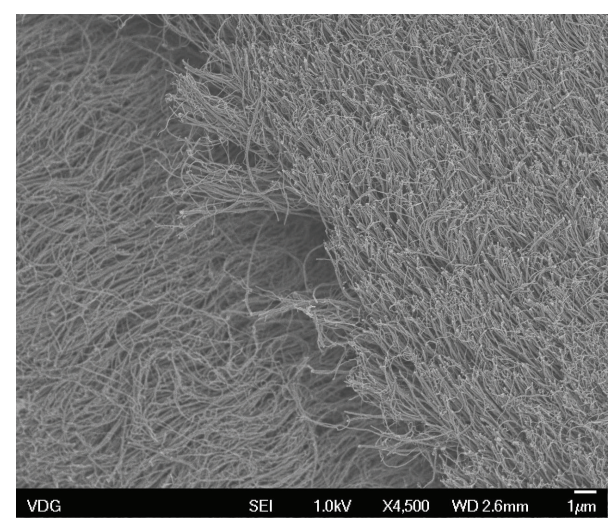

(a)

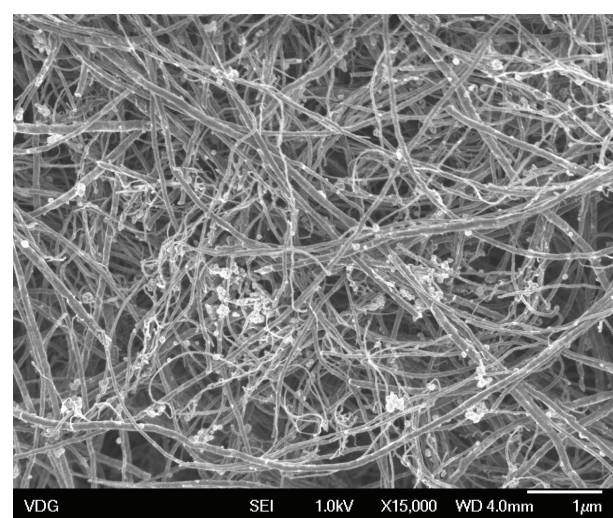

(b)

FIGURE 1: (a) SEM image of a pure MWCNTs sample. (b) SEM image of MWCNTs decorated with silver nanoparticles [(Ag/C) $0=0.88]$.

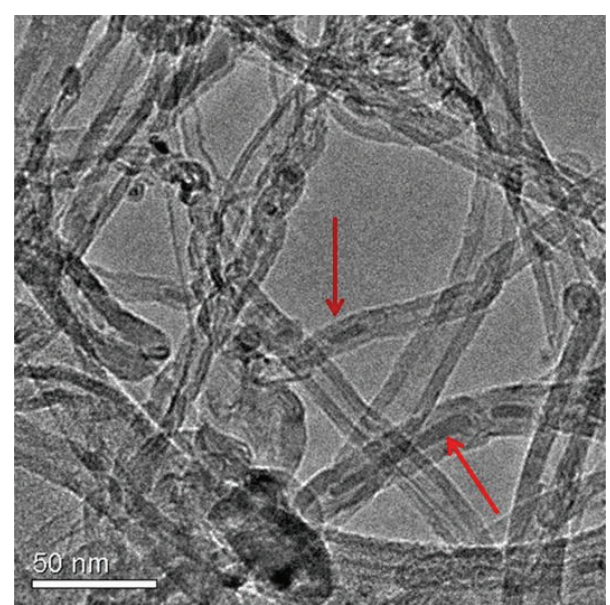

FIGURE 2: TEM image of pristine MWCNTs. The arrows indicate the position of iron catalyst nanoparticles.

advantage of producing high yields of relatively clean nanotubes via the atomization of a solution released from a liquid reservoir through a preheated quartz tube. A solution of ferrocene $\left(\mathrm{Fe}\left(\mathrm{C}_{5} \mathrm{H}_{5}\right)_{2}\right)$ in toluene $\left(\mathrm{C}_{7} \mathrm{H}_{8}\right)$ at a concentration of $2.3 \mathrm{wt} \%$ was used as the precursor liquid. This solution was pyrolyzed at $850^{\circ} \mathrm{C}$ and the obtained MWCNTs are as much as to tens of micrometers long with external diameters between 30 and $60 \mathrm{~nm}$.

For the decoration process, a stable homogenous MWCNTs suspension was obtained by dispersing $25 \mathrm{mg}$ of pristine MWCNTs into $1.0 \mathrm{wt} \%$ of sodium dodecyl sulfate (SDS) aqueous solution and sonicated for one hour combining a tip sonicator with an ultrasonic bath. The resulting MWCNTs suspension was introduced into Tollen's reagent formed by drop wise addition of $5 \%$ of ammonia to a silver nitrate $\left(\mathrm{AgNO}_{3}\right)$ aqueous solution under stirring until the brown precipitate just dissolved. Formaldehyde $(0.5 \mathrm{~mL})$ was dropped into the system and the system was maintaining stirring at $60^{\circ} \mathrm{C}$ for $45 \mathrm{~min}$. The final silver-functionalized MWCNTs were collected by centrifugation and washed with water and ethanol several times. The obtained black powder was dried at room temperature for $24 \mathrm{~h}$. Three different concentrations of silver nitrate solutions were prepared such that the initial silver to carbon stoichiometry was fixed to $(\mathrm{Ag} / \mathrm{C})_{0}=0.88,(\mathrm{Ag} / \mathrm{C})_{0}=0.44$, or $(\mathrm{Ag} / \mathrm{C})_{0}=0.22$.

2.2. Characterization. The samples were dispersed in isopropanol, and a few droplets of the suspension were transferred onto a holey carbon grid for TEM and HREM observation and analysis. High-resolution transmission electron microscopy was performed using a JEOL TEM JEM-3010 equipped with a $\mathrm{LaB}_{6}$ filament and a high-resolution pole piece $(\mathrm{Cs}=0.5 \mathrm{~mm})$. The microscope operated at $300 \mathrm{kV}$ for high-resolution imaging $(0.17 \mathrm{~nm})$ (HREM). Scanning electron microscopy was performed using a JEOL JSM-6701F field emission scanning electron microscope.

$\mathrm{X}$-ray diffraction (XRD) measurements were performed on a D8 ADVANCE $\mathrm{X}$-ray diffractometer using $\mathrm{Cu} \mathrm{K}_{\alpha}$ radiation $(\lambda=1.54 \AA$ ) while the $\mathrm{X}$-ray photoelectron spectroscopy (XPS) was performed using a spectrometer equipped with a commercial hemispherical electron energy analyzer Alpha 110 with $\mathrm{Al} \mathrm{K} \alpha$ radiation $(h v=1486.58 \mathrm{eV})$. The XPS spectra were collected at a fixed analyzer pass energy of $20 \mathrm{eV}$ and the data were processed using the CasaXPS software. The quantitative $\mathrm{Ag} / \mathrm{C}$ atomic ratios of the samples were determined using the peak area ratio of the corresponding XPS core levels and the sensitivity factor of each element in XPS.

Raman spectroscopy analysis was performed using a micro-Raman spectrometer (NT-MDT, NTEGRA SPECTRA) equipped with a CCD detector and an excitation solid state laser with wavelength of $473 \mathrm{~nm}$.

\section{Results and Discussion}

MWCNTs were decorated by nanoparticles using the silver mirror reaction. Different concentrations of solutions were prepared such that the initial $\mathrm{Ag}$ to $\mathrm{C}$ atomic ratio $(\mathrm{Ag} / \mathrm{C})_{0}$ can be varied. The pure multiwalled carbon nanotubes obtained by decomposition of a solution containing $2.3 \mathrm{wt} \%$ ferrocene in toluene are shown in Figure 1(a). The typical 


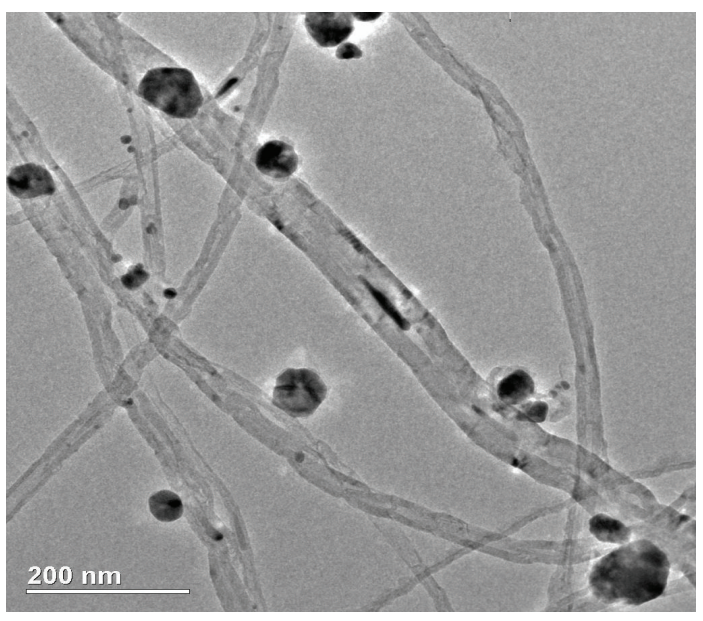

(a)

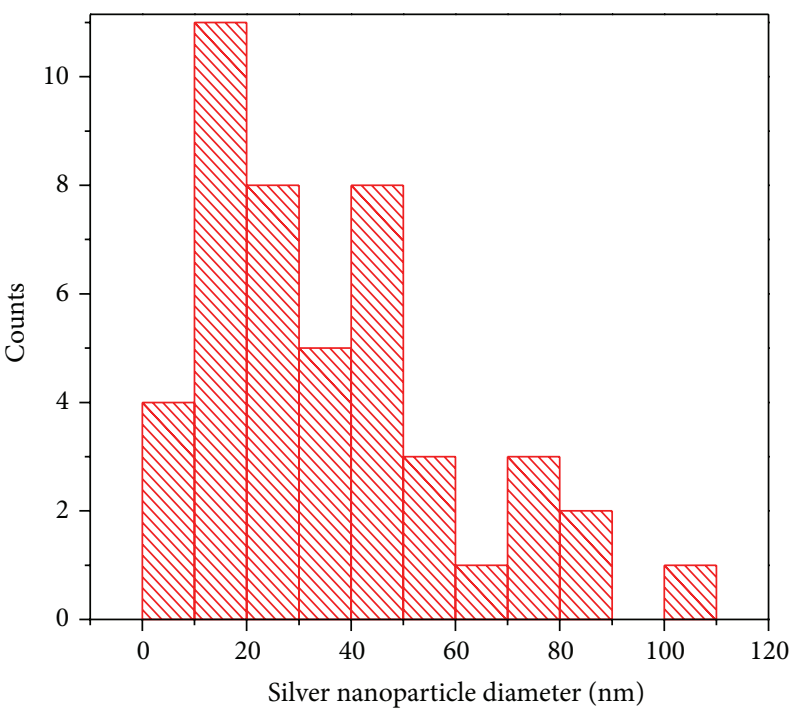

(c)

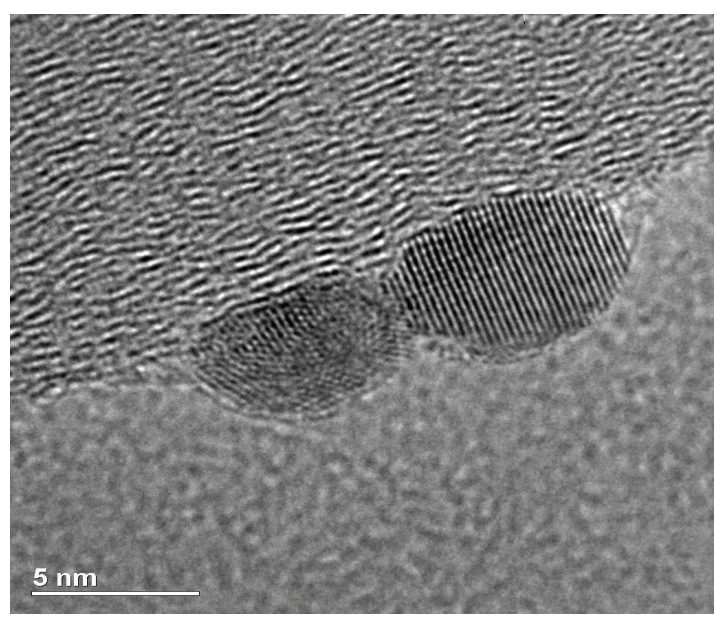

(b)

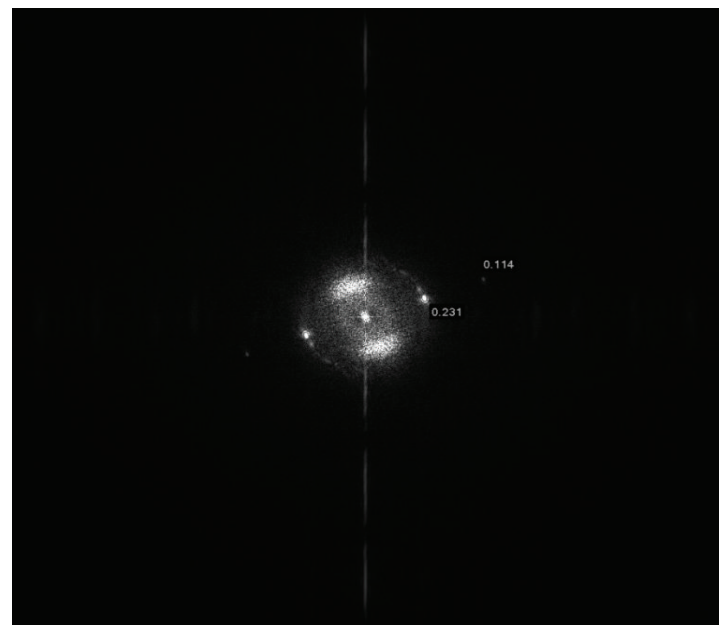

(d)

FIGURE 3: (a) TEM image of representative coated tubes with Ag nanoparticles. (b) HRTEM image showing Ag nanoparticles attached to the tube wall. (c) Ag nanoparticles size distribution obtained from TEM images. (d) FFT of the image obtained from the region shown in (b) containing the larger nanoparticle and the outer layers of the MWCNT. The image shows the graphitic planes $(0.34 \mathrm{~nm}$ interplanar distance) and silver planes ( $0.23 \mathrm{~nm}$ interplanar distances) perpendicular to each other [25]. Sample prepared with initial Ag to C atomic ratio, $(\mathrm{Ag} / \mathrm{C})_{0}=0.44$.

length of the tubes is in the range of $5-6 \mu \mathrm{m}$, while the average external diameters range from 30 to $60 \mathrm{~nm}$.

Pristine multiwalled CNTs image obtained by TEM was show in Figure 2. MWCNTs tend to maintain their crystalline structure, despite the damage induced by high energy beam used for the analysis. The catalyst iron nanoparticles were clearly showed inside the tubes and indicated by arrows.

Subsequently, these nanotubes were decorated with silver nanoparticles. The silver nanoparticles were formed by addition of the solution containing nanotubes to the Tollen's reagent [24]. The resulting material is shown in Figure 1(b) in a representative SEM image of the decorated tubes. The silver nanoparticles are the bright spots on the CNT surfaces. The image indicates that the nanoparticles are fairly homogeneously distributed on the CNTs surface.
Representative TEM images and HRTEM images of the nanocomposite are shown in Figure 3. The samples were dispersed in isopropanol, and a few droplets of the suspension were transferred onto a holey carbon grid for transmission electron microscopy observation and analysis. TEM image shown in Figure 3(a) exhibits silver particles with sizes ranging from 20 to $80 \mathrm{~nm}$ attached on their outer layer. Almost no free particles were observed in the background of the TEM images, which confirms that most of the formed nanoparticles were firmly anchored to the nanotubes.

HRTEM was used to determine how silver nanoparticles were attached to the surface of the nanotubes. The shape and crystallographic structure of the silver nanoparticles with respect to the graphite planes in the tubes are visible in the HRTEM images presented in Figure 3(b). The nanocrystals 
growing on the tube surface apparently did not damage the nanotube wall structures. In fact, the images clearly show that the parallel layers maintain the $0.34 \mathrm{~nm}$ interplanar distances of the MWCNT. In fact, in Figure 3(d) the fast Fourier transform (FFT) of the phase contrast image at the interface between the tube and nanoparticle is presented [25]. It reveals (0002) graphitic planes $(0.34 \mathrm{~nm}$ interplanar distance) and (111) silver planes (0.23 nm interplanar distances) perpendicular to each other. In Figure 3(c), we show the nanoparticles size distribution obtained from several TEM images taken from different regions of the same sample. This is a broad distribution centered at around $30 \mathrm{~nm}$. The larger particles (sizes $>100 \mathrm{~nm}$ ) probably are aggregation of smaller ones. It is important to call the attention to the fact that pristine MWCNTs when decorated with metallic nanoparticles usually presented a broad distribution when compared with the one obtained for nitrogen-doped MWCNTs. It was observed before for nickel particles [26] and confirmed for Ag nanoparticles [27]. However, the results reported in [25] do not agree with ours, since they claimed that only $\mathrm{N}$-doped MWCNTs exhibit a uniform coverage with Ag nanoparticles while using pristine MWCNTs results in an inhomogeneous coverage of Ag clusters that tend to agglomerate with other Ag particles. Our TEM results show that this agglomeration occurs; probably it is responsible for the larger sizes observed in the distribution shown in Figure 3(c) but also indicates that the nanoparticles are homogeneously distributed on the CNTs surface.

The XRD pattern of the composite sample prepared using the initial stoichiometry $(\mathrm{Ag} / \mathrm{C})_{0}=0.44$ is shown in Figure 4. The diffraction peak at $2 \theta=26.2^{\circ}$ is assigned to the (002) plane of the MWCNTs. The position of the other characteristic peaks in Figure 4 corresponds to a silver face centered cubic structure. The diffraction angles at $2 \theta=$ $38.2^{\circ}$ and $44.5^{\circ}$ can be assigned to the (111) and (200) planes of the Ag crystal lattices, respectively. In XRD, the size of coherently diffracting domains is measured and it does not necessarily agree with the crystalline grain size. The average crystallite size of the silver nanoparticles, which was calculated using the Debye-Scherer formula [28], was determined to be approximately $30 \mathrm{~nm}$, which is consistent with the size of the silver nanostructures observed by electron microscopy. No significant dependence on the concentration of the silver nitrate aqueous solution, $(\mathrm{Ag} / \mathrm{C})_{0}$, was observed.

XPS analysis was performed to elucidate the surface state composition of the Ag-MWCNTs nanocomposite. The full spectrum showed in Figure 5(a) reveals the dominance of silver and carbon, with small amounts of sodium and sulfur in the sample. The presence of $\mathrm{Na}$ and $\mathrm{S}$ originates from the use of sodium dodecyl sulfate (SDS) for the MWCNTs dispersion. The oxygen content increases compared to a pure MWCNTs sample. However, there is no evident relationship between the oxygen and the silver contents because the O/Carbon atomic rate did not change significantly between the different silver concentrations.

The spectrum of the Ag 3d core level of the Ag-decorated MWCNTs, which is shown in Figure 5(b), clearly confirms the presence of metallic silver because the $3 \mathrm{~d}_{5 / 2}$ component

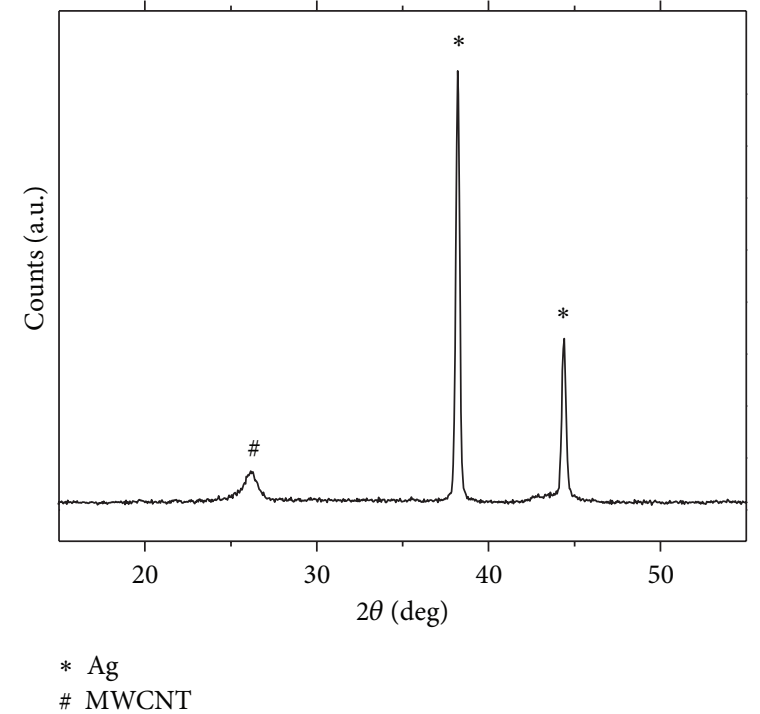

FIGURE 4: X-ray diffraction pattern taken of the composite sample prepared with initial $\mathrm{Ag}$ to $\mathrm{C}$ atomic ratio, $(\mathrm{Ag} / \mathrm{C})_{0}$, of 0.44 .

occurs at a binding energy of $368.3 \mathrm{eV}$, which is characteristic of the metallic $\mathrm{Ag}(0)$ oxidation state.

Because the photoelectron's free mean path on the material is smaller than the mean nanotube radius and larger than the mean silver particles diameter, the calculated Ag/C atomic ratio obtained from XPS spectrum is an upper bound. In Figure 6, we have shown the XPS spectra of Ag 3d core level obtained from different samples. The decoration efficiency can be defined as $\left[(\mathrm{Ag} / \mathrm{C})_{\mathrm{XPS}} /(\mathrm{Ag} / \mathrm{C})_{0}\right]$ as a function of the initial silver stoichiometry $(\mathrm{Ag} / \mathrm{C})_{0}$. The calculated efficiencies are $2 \%$ for the $(\mathrm{Ag} / \mathrm{C})_{0}=0.88,4 \%$ for the $(\mathrm{Ag} / \mathrm{C})_{0}=0.44$, and $2 \%$ for the $(\mathrm{Ag} / \mathrm{C})_{0}=0.22$ samples.

The main features in the Raman spectra shown in Figure 7 are the $D$ band at around $1350 \mathrm{~cm}^{-1}$ and the $G$ band at $1580 \mathrm{~cm}^{-1}$. A second order band at $2750 \mathrm{~cm}^{-1}$ was also observed. Raman spectroscopy is frequently employed to probe the quality of carbon nanotubes. The ratio between the intensities of the $D$ and $G$ bands, $I_{D} / I_{G}$, is a parameter commonly used for this purpose [29]. In Figure 7, we show the Raman spectrum obtained from pure MWCNTs. This spectrum has a low $I_{D} / I_{G}$ value, (i.e., 0.23 ), which is typical of good quality nanotubes. In the same figure, we show Raman spectra of MWCNTs decorated with silver nanoparticles. The positions of the bands are essentially the same, while the $I_{D} / I_{G}$ slightly increased upon the increase of silver nanoparticles attached to the nanotubes surfaces. However, the low $I_{D} / I_{G}$ values indicate that the adopted procedure preserved the integrity of the MWCNTs, confirming the HRTEM results.

A wet-chemical route to obtained silver nanoparticlesCNT composites was already published [17]. Despite some differences in the experimental route to prepare the composite, in particular the way used to disperse the MWCNTs, our results are in general agreement with the previous one. However, in the present work we present an accurate analysis 


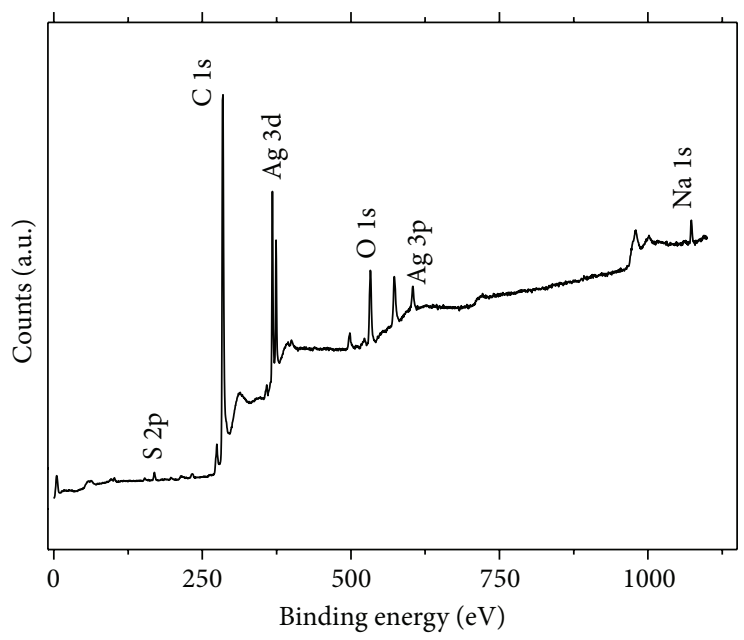

(a)

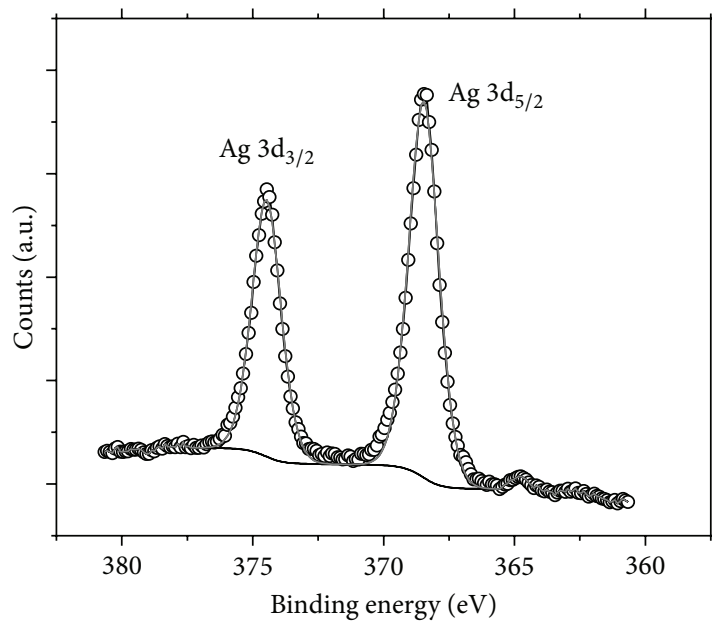

(b)

FIGURE 5: (a) XPS spectrum of the silver nanoparticles/MWCNTs composite prepared with initial Ag to C atomic ratio, (Ag/C) $)_{0}$, of 0.44 . (b) XPS spectrum of Ag $3 \mathrm{~d}$ core level of same sample. The spectrum was fitted taking into account the Shirley background.

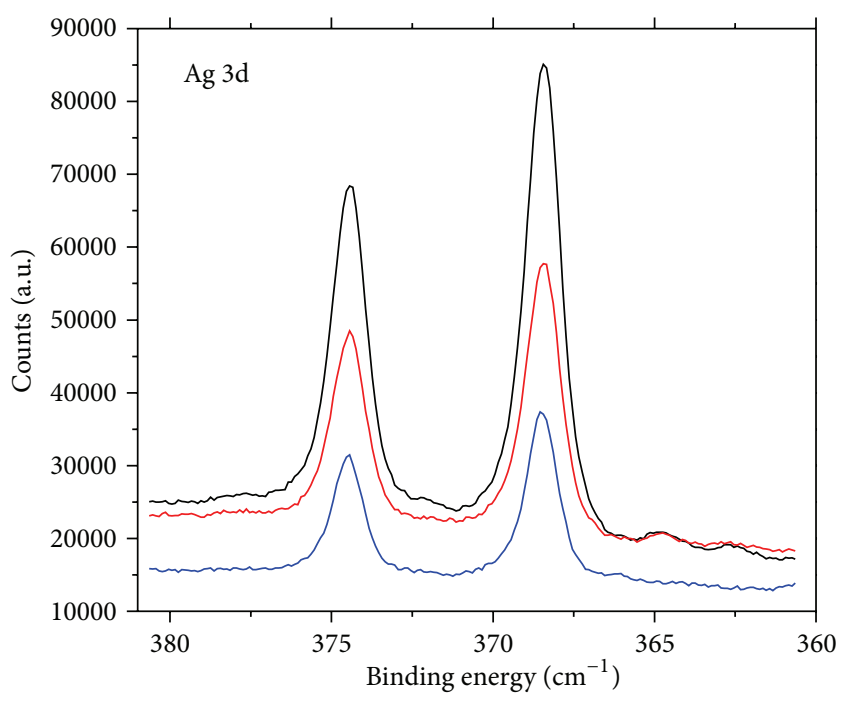

$(\mathrm{Ag} / \mathrm{C})_{0}=0.88$

$(\mathrm{Ag} / \mathrm{C})_{0}=0.44$

$(\mathrm{Ag} / \mathrm{C})_{0}=0.22$

FIGURE 6: XPS spectra of Ag 3d core level of composite samples prepared with different initial $\mathrm{Ag}$ to $\mathrm{C}$ atomic ratio $(\mathrm{Ag} / \mathrm{C})_{0}$.

of TEM results showing the crystallographic orientation of the particles with respect the MWCNTs surface, as well as the nanoparticles size distribution.

Other simple routes were also used to decorate MWCNTs with silver nanoparticles $[18,30]$. The photochemical deposition of Ag nanoparticles on MWCNTS produces particles with average size at around $21 \mathrm{~nm}$ and the authors claimed that using this technique they can control the average size of the particles [18], while another simple chemical route produces Ag-MWCNTs composite with particles with an average size of $18 \mathrm{~nm}$ [30].

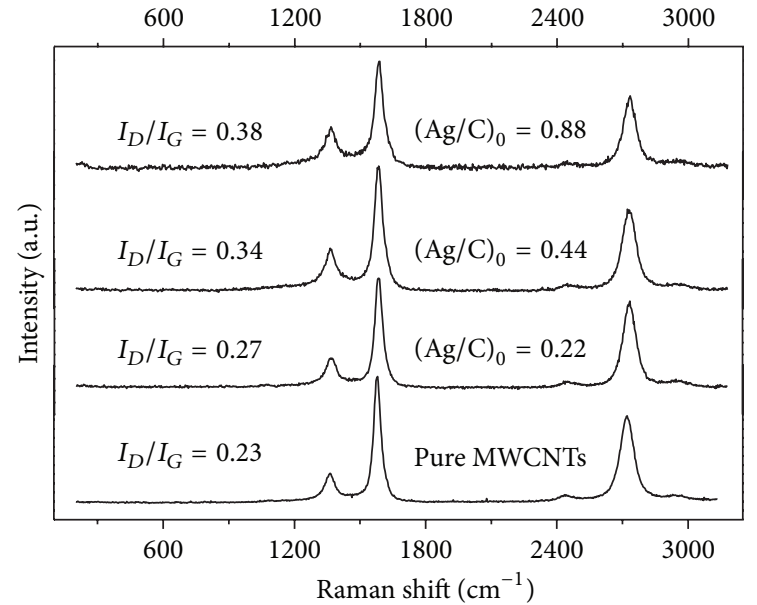

FIGURE 7: Raman spectra of Ag nanoparticle-MWCNTs composites prepared with different initial $(\mathrm{Ag} / \mathrm{C})_{0}$ ratios.

\section{Conclusions}

In this work, we studied the decoration of MWCNTs synthesized via spray pyrolysis with silver nanoparticles through a simple wet chemistry route that was based on the silver mirror reaction. A multitechnique approach was adopted for the samples characterization. The nanoparticles were found to be spatially well dispersed on the carbon nanotubes. HRTEM revealed the orientations of the crystallography planes of the anchored silver nanoparticles and indicated that the (111) plane of silver face centered cubic structure is perpendicular to the (0002) graphitic planes of the MWCNTs. This information can be important for modeling the process of nucleation and growth of the nanoparticles on the nanotubes surfaces. 
The Raman results indicate that when the initial Ag to $\mathrm{C}$ atomic ratio $(\mathrm{Ag} / \mathrm{C})_{0}$ increases, the nanotube integrity was preserved. We also studied the silver content as a function of the initial $(\mathrm{Ag} / \mathrm{C})$ atomic ratio by means of photoelectron spectroscopy. These results showed that the efficiency of the composite formation process is, within experimental uncertainties, nearly independent of $(\mathrm{Ag} / \mathrm{C})_{0}$. The metallic character of the silver nanoparticles is independent of the different initial $(\mathrm{Ag} / \mathrm{C})$ atomic ratio, as demonstrated by XPS analysis.

Silver-MWCNTs composite with particle size distribution centered at around $20-30 \mathrm{~nm}$ is suitable for several applications as catalysts or sensing materials because of the appropriate surface to volume ratio of the particles $[4,10,11$, 14]. The procedure discussed in this work permits a simple way to obtain this composite controlling the number of particles by changing the initial Ag to $\mathrm{C}$ atomic ratio.

\section{Conflict of Interests}

The authors declare that there is no conflict of interests regarding the publication of this paper.

\section{Acknowledgments}

The authors wish to thank Mr. Fernando Henrique Monteiro and Mr. Erick Talarico for help during sample preparation and analysis. This research was supported by the Brazilian agencies $\mathrm{CNPq}$, CAPES, and FAPERJ. This work was also supported by the Brazilian Synchrotron Light Laboratory (LNLS) under Proposal TEM-HR-10269.

\section{References}

[1] V. Georgakilas, D. Gournis, V. Tzitzios, L. Pasquato, D. M. Guldi, and M. Prato, "Decorating carbon nanotubes with metal or semiconductor nanoparticles," Journal of Materials Chemistry, vol. 17, no. 26, pp. 2679-2694, 2007.

[2] J. R. Siqueira Jr., C. F. Werner, M. Bäcker et al., "Layer-bylayer assembly of carbon nanotubes incorporated in lightaddressable potentiometric sensors," Journal of Physical Chemistry C, vol. 113, no. 33, pp. 14765-14770, 2009.

[3] X. S. Wang, H. C. Wang, Y. J. Huang et al., "Noncovalently functionalized multi-wall carbon nanotubes in aqueous solution using the hydrophobin HFBI and their electroanalytical application," Biosensors and Bioelectronics, vol. 26, no. 3, pp. 1104-1108, 2010.

[4] Y. K. Yang, S. Q. Qiu, C. G. He, W. J. He, L. Yu, and X. Xie, "Green chemical functionalization of multiwalled carbon nanotubes with poly( $\varepsilon$-caprolactone) in ionic liquids," Applied Surface Science, vol. 257, no. 3, pp. 1010-1014, 2010.

[5] G. Wei, C. J. Pan, J. Reichert, and K. D. Jandt, "Controlled assembly of protein-protected gold nanoparticles on noncovalent functionalized carbon nanotubes," Carbon, vol. 48, no. 3 , pp. 645-653, 2010.

[6] D. G. Larrude, P. Ayala, M. E. H. Maia Da Costa, and F. L. Freire Jr., "Multiwalled carbon nanotubes decorated with cobalt oxide nanoparticles," Journal of Nanomaterials, vol. 2012, Article ID 695453, 5 pages, 2012.
[7] M. Scarselli, L. Camilli, P. Castrucci et al., "In situ formation of noble metal nanoparticles on multiwalled carbon nanotubes and its implication in metal-nanotube interactions," Carbon, vol. 50, no. 3, pp. 875-884, 2012.

[8] D. G. Larrude, M. E. H. Maia Da Costa, F. H. Monteiro, A. L. Pinto, and F. L. Freire Jr., "Characterization of phosphorusdoped multiwalled carbon nanotubes," Journal of Applied Physics, vol. 111, no. 6, Article ID 064315, 2012.

[9] Z. Chen, Z. Guan, M. Li, Q. Yang, and C. Li, "Enhancement of the performance of a platinum nanocatalyst confined within carbon nanotubes for asymmetric hydrogenation," Angewandte Chemie, vol. 50, no. 21, pp. 4913-4917, 2011.

[10] S. Sahoo, S. Husale, S. Karna, S. K. Nayak, and P. M. Ajayan, "Controlled assembly of Ag nanoparticles and carbon nanotube hybrid structures for biosensing," Journal of the American Chemical Society, vol. 133, no. 11, pp. 4005-4009, 2011.

[11] K.-S. Kim and S.-J. Park, "Influence of silver-decorated multiwalled carbon nanotubes on electrochemical performance of polyaniline-based electrodes," Journal of Solid State Chemistry, vol. 184, no. 10, pp. 2724-2730, 2011.

[12] O. Akhavan, M. Abdolahad, Y. Abdi, and S. Mohajerzadeh, "Silver nanoparticles within vertically aligned multi-wall carbon nanotubes with open tips for antibacterial purposes," Journal of Materials Chemistry, vol. 21, no. 2, pp. 387-393, 2011.

[13] A. B. Castle, E. Gracia-Espino, C. Nieto-Delgado, H. Terrones, M. Terrones, and S. Hussain, "Hydroxyl-functionalized and $\mathrm{N}$-doped multiwalled carbon nanotubes decorated with silver nanoparticles preserve cellular function," ACS Nano, vol. 5, no. 4, pp. 2458-2466, 2011.

[14] Y. Aimin, Q. Wang, J. Yong et al., "Silver nanoparticle-carbon nanotube hybrid films: preparation andelectrochemical sensing," Electrochimica Acta, vol. 74, pp. 111-116, 2012.

[15] H. T. Chung, J. H. Won, and P. Zelenay, "Active and stable carbon nanotube/nanoparticle composite electrocatalyst for oxygen reduction," Nature Communications, vol. 4, p. 1922, 2013.

[16] A. P. Alivisatos, "Semiconductorclusters, nanocrystals, andquantumdots," Science, vol. 271, no. 5251, pp. 933-937, 1996.

[17] Y. Liu, J. Tang, X. Chen, W. Chen, G. K. H. Pang, and J. H. Xin, "A wet-chemical route for the decoration of CNTs with silver nanoparticles," Carbon, vol. 44, no. 2, pp. 381-383, 2006.

[18] N. Tanaka, H. Nishikiori, S. Kubota, M. Endo, and T. Fuji, "Photochemical deposition of Ag nanoparticles on multiwalled carbon nanotubes," Carbon, vol. 47, no. 11, pp. 2752-2760, 2009.

[19] X. Hou, L. Wang, and R. Wu, "In-situ synthesis of highly dispersed silver nanoparticles on multiwalled carbon," Bulletin of the Korean Chemical Society, vol. 32, no. 8, pp. 2527-2528, 2011.

[20] Y. Jiang, Y. Lu, L. Zhang, L. Liu, Y. Dai, and W. Wang, "Preparation and characterization of silver nanoparticles immobilized on multiwalled carbon nanotubes by poly(dopamine) functionalization," Journal of Nanoparticle Research, vol. 14, p. 938, 2012.

[21] O. Akhavan, M. Abdolahad, Y. Abdi, and S. Mohajerzadeh, "Silver nanoparticles within vertically aligned multi-wall carbon nanotubes with open tips for antibacterial purposes," Journal of Materials Chemistry, vol. 21, no. 2, pp. 387-393, 2011.

[22] X. Chen, U. C. Tam, J. L. Czlapinski et al., "Interfacing carbon nanotubes with living cells," Journal of the American Chemical Society, vol. 128, no. 19, pp. 6292-6293, 2006.

[23] A. B. Castle, E. Gracia-Espino, C. Nieto-Delgado, H. Terrones, M. Terrones, and S. Hussain, "Hydroxyl-functionalized and $\mathrm{N}$-doped multiwalled carbon nanotubes decorated with silver 
nanoparticles preserve cellular function," ACS Nano, vol. 5, no. 4, pp. 2458-2466, 2011.

[24] L. Qu and L. Dai, "Novel silver nanostructures from silver mirror reaction on reactive substrates," Journal of Physical Chemistry B, vol. 109, no. 29, pp. 13985-13990, 2005.

[25] D. R. G. Mitchell, "DiffTools: electron diffraction software tools for DigitalMicrograph," Microscopy Research and Technique, vol. 71, no. 8, pp. 588-593, 2008.

[26] P. Ayala, F. L. Freire Jr., L. Gu et al., "Decorating carbon nanotubes with nanostructured nickel particles via chemical methods," Chemical Physics Letters, vol. 431, no. 1-3, pp. 104109, 2006.

[27] A. Zamudio, A. L. Elías, J. A. Rodríguez-Manzo et al., "Efficient anchoring of silver nanoparticles on n-doped carbon nanotubes," Small, vol. 2, no. 3, pp. 346-350, 2006.

[28] H. P. Klug and L. E. Alexander, X-Ray Diffraction Procedure, Wiley, New York, NY, USA, 2nd edition, 1974.

[29] M. A. Pimenta, G. Dresselhaus, M. S. Dresselhaus, L. G. Cançado, A. Jorio, and R. Saito, "Studying disorder in graphitebased systems by Raman spectroscopy," Physical Chemistry Chemical Physics, vol. 9, no. 11, pp. 1276-1291, 2007.

[30] K. Dai, L. Shi, J. Fang, and Y. Zhang, "Synthesis of silver nanoparticles on functional multi-walled carbon nanotubes," Materials Science and Engineering A, vol. 465, no. 1-2, pp. 283286, 2007. 

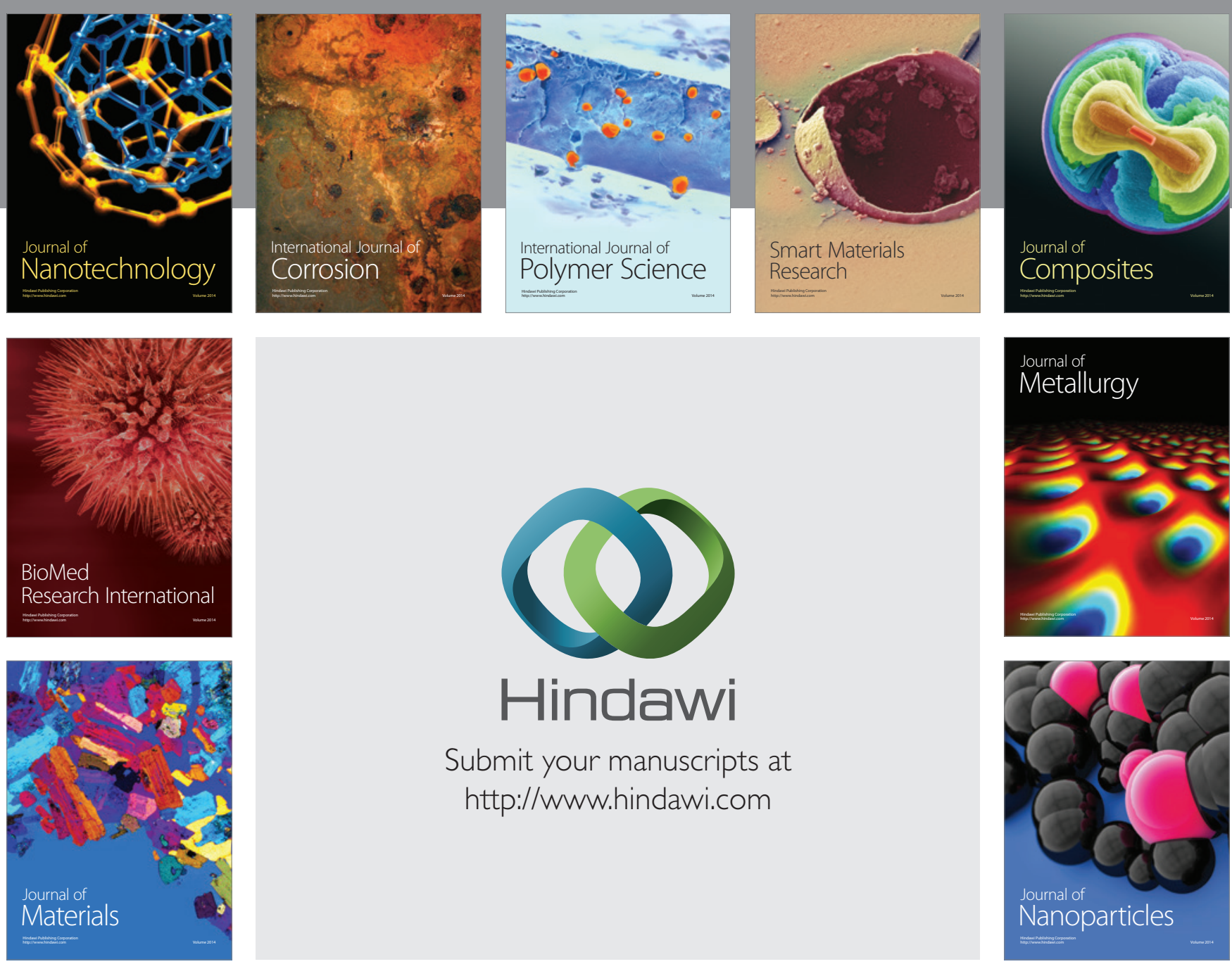

Submit your manuscripts at http://www.hindawi.com
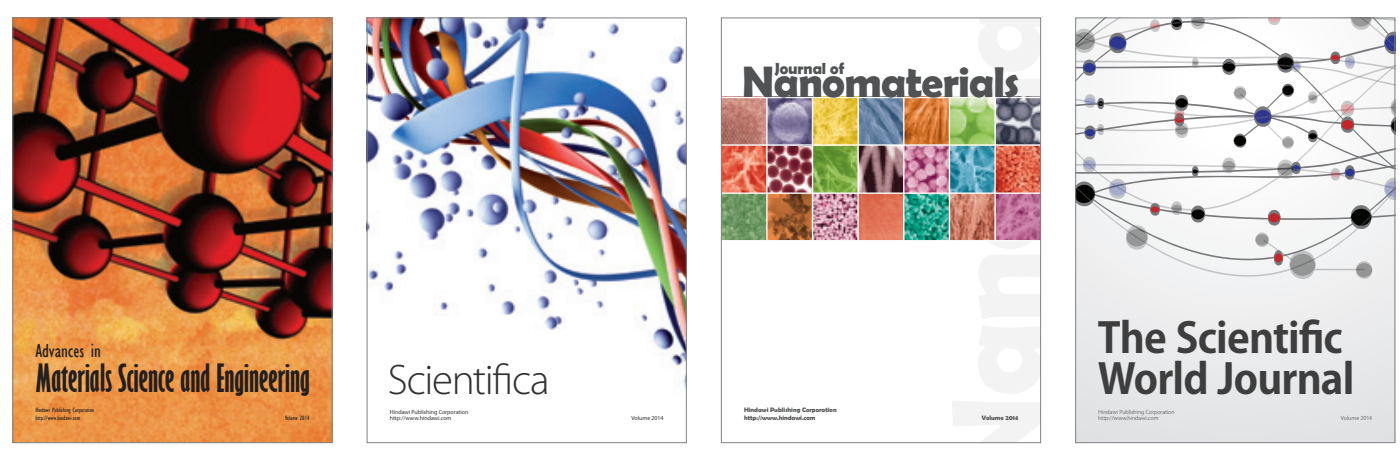

\section{The Scientific World Journal}
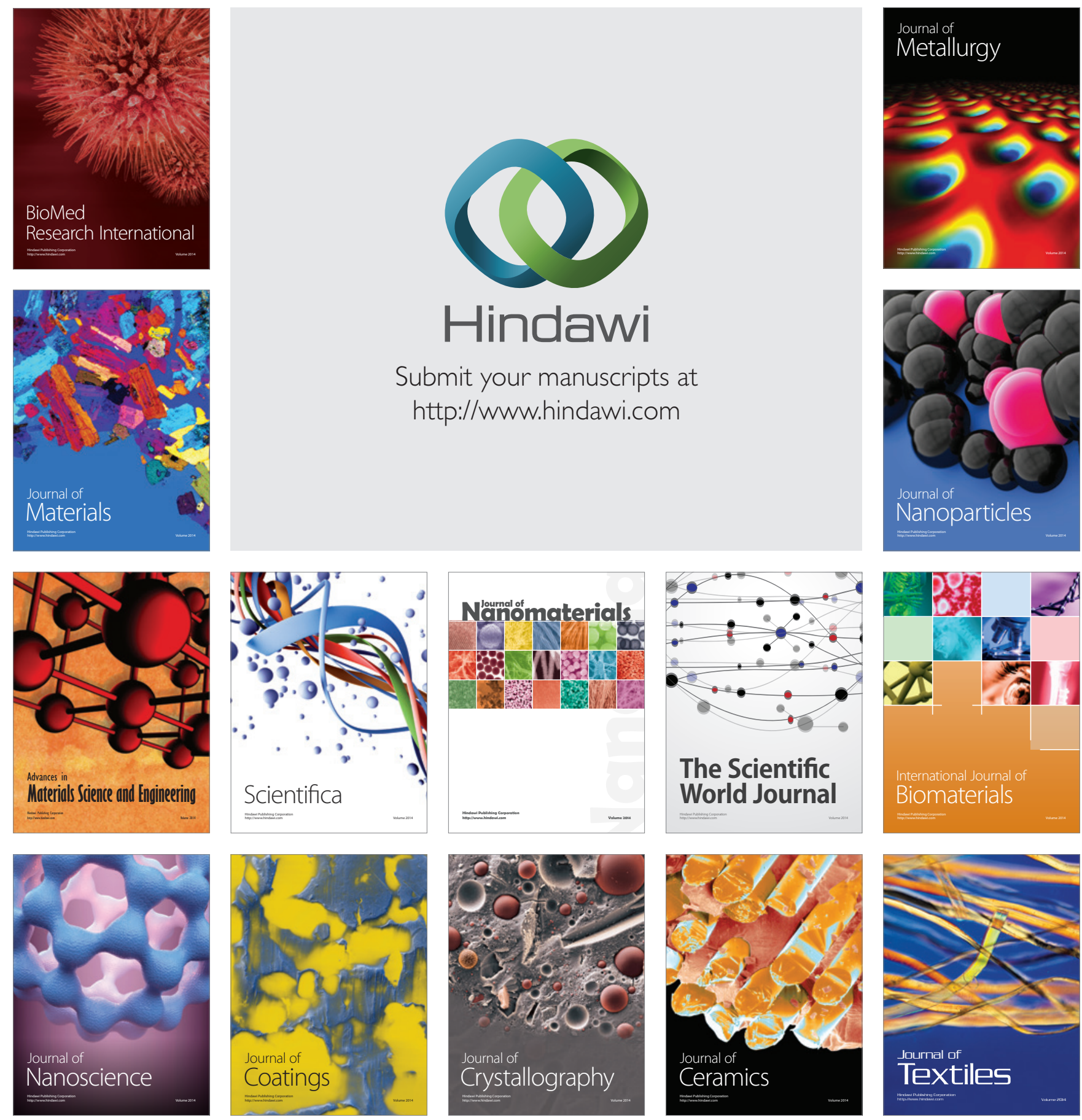